Running head: FACEBOOK AS DATA

\title{
Facebook as Data: A Primer for Educational Technology Researchers
}

\author{
Joshua M. Rosenberg \\ University of Tennessee, Knoxville
}

Author note. Correspondence regarding this article can be addressed to Joshua M. Rosenberg, jmrosenberg@utk.edu, 1122 Volunteer Blvd., Knoxville, TN 37996.

The analytic code and other files (except for the historical data, which must be accessed through CrowdTangle) is available at https://osf.io/jhnrb/?view_only=43f2da07eee14feb90fcb1755bbdbcfe. 


\begin{abstract}
Facebook is widely used and researched. However, though digital trace data has been used to study educational technology tools and other social media platforms, it has not been used very much to study Facebook. Instead, the same self-report surveys used in many educational research studies have been predominant. This gap concerning how Facebook has been examined may be significant given what has been learned from researchers using data mining or learning analytics approaches to analyze digital trace data at a massive scale. While it has been impractical to use Facebook as a data source, the CrowdTangle platform provides access to researchers - faculty and graduate students - to freely access the massive collection of posts on public Facebook pages and groups. In the absence of suitable examples or a tutorial, this paper provides a primer that offered some considerations for researchers before collecting data (i.e., conducting research ethically and framing the study) as well as those that directly pertain to using CrowdTangle: accessing the CrowdTangle platform, uploading or identifying pages (or groups), and downloading historical data. Lastly, this paper describes the steps taken to analyze the data to answer two research questions, with the intent of illustrating how the data could be used (and not to exemplify this process). To provide other researchers with a starting point for using the $\mathrm{R}$ statistical software and programming language by providing an $\mathrm{R}$ Markdown document with all the steps necessary to reproduce these analyses is provided. I discuss some directions for research that uses Facebook as a data source to understand educational topics and people and organizations.
\end{abstract}


Many people use Facebook: 69\% of American adults say they ever use it based on the latest polling from Pew Research, with 49\% using it several times per day (Gramlich, 2021). No other platform apart from YouTube is used by as many Americans, and apart from Pinterest, no other online platform has a smaller politically partisan divide (Gramlich, 2021). In addition to being widely used, Facebook has been widely researched - including the use of Facebook in educational settings (Barrot, 2021; Chugh \& Ruhi, 2018; Greenhow et al., 2020; Niu, 2019).

However, though digital trace data has been used to study educational technology tools and other social media platforms, it has not been used very much to study Facebook. Instead, studies have found that the same self-report surveys used in many educational research studies have been predominant (Niu, 2019). The absence of research using digital trace data is notable given how scholars across educational research have used this type of data (Fischer et al., 2020). Moreover, sub-fields of educational research, including the learning analytics and educational data mining communities, have shown how digital platforms can study teaching, learning, and educational systems (Siemens \& Baker, 2012; Wise, 2019).

Until recently, it has been difficult (and, at times, not possible) to use Facebook as a data source, but there is now a platform that provides access to Facebook data at a massive scale. In this paper, I argue for the potential value of using Facebook as a data source for educational technology research. After this, I provide an accessible tutorial for faculty and graduate students seeking to use Facebook data for their research.

\section{Using Digital Trace Data for Research}

Systematic reviews have shown that much of the research on the educational uses of Facebook has relied on a single research approach: self-report surveys (Niu, 2019). This issue is not exclusive to Facebook: Greenhow et al. (2020) also argued that self-report survey methods are shared across research on K-12 teachers' use of social media. At the same time, Greenhow et al. note that other methods, both more traditional and newer (i.e., public data mining), have complemented studies based on teachers' self-reports of their experiences with social media or the benefits they achieve from their use. Notably, the use of what scholars have termed digital trace data (Hakimi et al., 2021) invite new means of studying social media platforms such as Facebook, mainly when this data is analyzed using methods suited to the analysis of large, complex data sets that often number in the millions of data points. For example, Carpenter et al. (2020) accessed data on millions of tweets posted to one or more of 16 education-related hashtags - after which they examined when, how, and by whom these hashtags were used.

The digital trace data pointed to in the review by Greenhow et al. (2020) are by no means only within the purview of a particular crop of researchers who focus on educational social media. For example, in addition to social media, educational researchers have used digital trace data when teachers and students use educational games and simulations (e.g., Gobert et al., 2013) and intelligent tutoring systems (e.g., Heffernan \& Heffernan, 2014), alongside many other tools and platforms, like discussion platforms and assessments (Fischer et al., 2020).

Given the features of Facebook and those of digital trace data, it is reasonable to assume that many studies have used Facebook as a source of data. Such studies might have yielded insights into the topics and events that are discussed, the people and organizations involved, and how platforms afford and constrain certain types of discussion or the involvement of actors. But this is predominantly not the case: While digital trace data has been used to study many features of other social media platforms - especially Twitter - few studies have studied Facebook using its data, instead of collecting data through users' self-reports (Niu. 2019). 
This gap in what research has been done may be important. We have learned how other platforms play a prominent role in education through studies that use large sets of digital trace data accessed through the platform; Facebook may play a similar role, but we do not know because Facebook has been closed to researchers. Furthermore, the exclusive focus on the platforms that have permitted access to researchers to data may introduce bias in terms of the nature of the explanations researchers develop and the representativeness of samples drawn from a single platform (Tufekci, 2014).

While using Facebook as a source of data was not possible in the wake of the company prohibiting the use of its data for any purpose following the unauthorized access by a private, third-party, Cambridge Analytica (Stewart, 2019), it is now possible for researchers (and journalists) to access Facebook data for research, reporting, and accounting through a platform that Facebook acquired, CrowdTangle ${ }^{1}$. This paper, then, provides a primer on how Facebook can be used for educational technology research by using newer public data mining methods (Kimmons et al., 2018) to access digital trace data from Facebook. To establish the value, I first review prior research on using digital trace data to study other social media platforms.

\section{Using Digital Trace Data to Study Platforms Other Than Facebook}

Many studies have focused on topics or events (e.g., Carpenter et al., 2020; Greenhow \& Gleason, 2014; Rosenberg et al., 2020; Staudt Willet, 2019). For example, Greenhalgh and Koehler (2017) studied how French educators prepared for and supported one another in the days following a terrorist attack using the \#educattentats hashtag (one that combined French words for education and terrorist attach). To do so, the authors used the Twitter Archiving Google Sheet; Hawksey, 2014) and web scraping of Twitter to access and record the content (or message) of every post and associated information, such as when the post was created and how many retweets and likes posts received. Using this data, the authors used qualitative methods and the aforementioned data mining methods to examine and report the purpose of the tweets, including the hashtag.

Many studies have also examined people or organizations (e.g., Gleason, 2016; RomeroHall, 2018; Trust et al., 2016). For example, Veletsianos and Kimmons (2016) used Twitter's Application Programming Interface (API) to programmatically (using code) identify all the individuals who posted one or more tweets to the hashtag for the 2014 American Educational Research Association (AERA) annual meeting. Then, the authors accessed the most recent 3,500 tweets sent by all those individuals. Then, the authors examined some key metrics that could signal the degree to which individuals are influential within educational research, finding that individuals identified as professors had more followers, for instance, but that a minimal amount of the variation in follower counts was strictly due to being a professor: many other factors other than one's professional role seems to matter on this platform.

Two themes cut across these groupings. First, even data mining studies using digital trace data often involve multiple methods, whether the authors consider their study to be a mixedmethods study. For example, Greenhalgh and Koehler (2014) and Veletsianos and Kimmons (2016) used qualitative methods in their studies. Also, we can see that studies have documented the specific affordances, the types of activity that are possible or easy to carry out (Kennewell, 2001; Tufekci, 2014), of platforms for researchers - and in some ways have been bound by them. For instance, Greenhalgh and Koehler (2014) could not determine who liked certain tweets, only that tweets received a particular number of likes. Furthermore, Veletsianos and Kimmons were

\footnotetext{
${ }^{1}$ https://www.crowdtangle.com/
} 
able to access the most recent 3,500 tweets of individuals; tweets posted further in the past could not be accessed.

\section{Studying the Educational Uses of Facebook Using Traditional and Newer Methods}

A recent review study found that four of the 15-most cited articles across all articles published a decade in 65 educational technology and instructional design were on Facebook (Bodily et al., 2019). Review papers on the use of social media in education have noted the prominence of research on this platform (and the social media platform Twitter; Barrot, 2021; Greenhow et al., 2020). A systematic review of prior research on the use of Facebook has shown that the platform can have educational benefits—-such as enhancing teacher-student interactions and improving students' academic achievement — as well as potential drawbacks — such as educators being more active on the platform than their students when Facebook is used as a part of courses (Chugh \& Ruhi, 2018).

As just noted, a systematic review on the use of Facebook has illustrated some of the positive (e.g., a lower barrier to students interacting with their teacher or instructor) and negative aspects (e.g., an imbalance in how active students and teachers are) of the use of the platform (Chugh \& Ruhi, 2018). However, reviews have also pointed to some of the methodological shortcomings of the extant research on Facebook: around two-thirds of the studies using experimental designs relied exclusively on self-report surveys, which may lead to biased findings of how positively students view the use of the platform as a part of their coursework (Niu, 2019).

Regarding the gaps in past research on Facebook, Niu (2019) points explicitly to content analysis as a methodology that could serve as a more valid source of data from which inferences about students' use of the platform could be made. A few studies have employed an approach or one like it, using posts to make inferences about the depth of conversations taking place among teachers in Facebook groups (Liljekvist et al., 2021; Lundin et al., 2020; van Bommel et al., 2020) or using posts as a measure of the depth of participation on the platform (Bowman \& Akcaoglu, 2014), for instance, but such uses are limited. Notably, these studies involve the analysis of a select sample of posts. Lundin et al., for instance, examined the linguistic differences in 79 posts receiving many comments or likes in a group focused on flipped classrooms. Liljekvist et al. and van Bommel et al. qualitatively coded 553 posts to one of six mathematics- or Swedish-language education-focused groups on Facebook, establishing the Pedagogical Content Knowledge (Liljekvist et al., 2021) and the depth of the interaction present in the discussions that took place in the context of posts and comments in response to posts in the groups. As noted, these studies have involved manually collecting data from the platform. In these cases, the researcher must manually access and screenshot or copy and paste posts and their content into a spreadsheet or document for subsequent analysis. In this way, studies have not taken advantage of one of the key affordances of digital trace data: it can be analyzed at a heretofore impractical scale (Greenhow et al., 2020).

Though limited, several studies have used digital trace data from Twitter in more automated (rather than manual-by hand-ways) that leverage the scale of social media data in a way that is different from and complementary to the more selective sampling approach used in other - predominantly qualitative - research. The work of Barnes and colleagues is especially noteworthy in this respect. Barnes (2021), for example, used the CrowdTangle platform to document interactions with media stories shared on public Facebook pages or in public Facebook groups about English-language teaching in Australia. Specifically, Barnes examined conversations and debates about the value of two English-language teaching (and reading) approaches, Universal Synthetic Phonics (or "phonics") and Whole Language, finding that 
advocates of the different approaches to teaching used social media to frame and shape the debate. Other studies have used CrowdTangle to access the contents of the posts of United States schools and school districts, finding that these educational institutions have posted an enormous number of messages: around 17 million through the end of 2020 (Burchfield et al., 2021). Research on these posts has illustrated the kinds of educational technologies schools and districts most often mention in posts (Kimmons et al., 2021, 2022), what schools and districts shared posted during the early phases of the COVID-19 pandemic (Rosenberg \& Nguyen, 2021), and how these posts may - inadvertently - threaten the privacy and safety of students given the ease with which photos linked to the names of students can be publicly accessed (Burchfield et al., 2021).

Next, I elaborate on how studies that focus on these two areas - topics and events and people and organizations - could be carried out.

\section{Primer: United States School Districts on Facebook}

If one is interested in examining the topics or events or people or organizations, how can one proceed? This section walks through the entire analysis process, from crafting a research question and gaining access to the platform through which one can access Facebook data to presenting the results using an open-source software tool, $R$ (R Core Team, 2021). Specifically, this section discusses the following six steps:

1. Conducting ethical research

2. Framing the research

3. Accessing CrowdTangle

4. Creating and uploading a list of pages to CrowdTangle

5. Analyzing data using R

\section{Conducting Ethical Research}

Instead of last, drawing on Greenhalgh et al.'s (2020) chapter, I consider ethics before framing a study and developing research questions. I summarize their advice here, pointing readers to that chapter for a more in-depth consideration of this topic. Greenhalgh et al. suggest first considering human subjects research-related consideration: just because social media data may be publicly accessible does not mean that ethics-related procedures - including Institutional Review Board approval — do not apply. But, often, analyses of social media are not strictly human subjects research, and it is not unreasonable to then think that ethical considerations matter less than they do in other research projects, but that may not be the case. Greenhalgh et al. point out that even research that is not within the purview of Institutional Review Board approval because it is not considered to be human subjects research should still be the focus of reflection and care - a point like one made by other educational technology researchers and writers (e.g., Kimmons et al., 2018; Krutka et al., 2019; Watters, 2014). Specific ethics-related principles for social media research selected by Greenhalgh et al. are to a) consider what is public and private on social media, b) balance harms and benefits, c) consider vulnerable individual groups, d) appropriately and carefully anonymize data, e) decide whether consent must be gained, and f) consider laws and terms of service.

\section{Framing the Research}

When one has access to social media data, the temptation may be to simply describe and present the data: The data is often rich and exciting - worthy of sharing. However, research can have a much greater impact when it is framed in light of particular ideas, theories, and findings from other research. Fundamentally social media research is not different from research on or with data generated from other contexts for research (like classrooms). Social media research 
requires careful framing. Greenhalgh et al. (2020) also select a number of considerations concerning how to frame social media research; I briefly summarize the considerations selected here: a) consider underlying worldviews and the assumptions one brings to research (i.e., what assumptions one holds or takes to be true about learning), b) articulate the research design and methods, c) use a conceptual framework - one particular to social media or educational technology research or not, and d) specify the target phenomena or unit of analysis.

For this tutorial, I consider research questions that are about topics or events and people or organizations. Questions may target each individually - or their intersection. For this tutorial, I will explore questions that are relatively simple and that have only a minimal bearing on the considerations for framing research I have described.

Pertaining to a topic or event, I will consider the days immediately before and during the closure of schools during the earliest stages of the COVID-19 pandemic. Our question is: When did districts first post about the COVID-19 pandemic? Related to people or organizations, we might be interested in variation in activity and interactions. Are the largest districts receiving more interactions? Those in cities? I ask: Which districts' pages received the most interactions throughout 2021? As already suggested, these are relatively straightforward questions, but answering them will touch on many of the aspects involved in a range of research studies involving the use of Facebook data.

\section{Accessing CrowdTangle}

Like many social media platforms and digital sources of data, it is necessary to first register or apply to access the data. While some websites allow you to register with a username and other information to access data, the platform used to access Facebook data, CrowdTangle, requires a brief application ${ }^{2}$ to screen for allowed use; Twitter has a very similar process.

As of early 2022, the application has the following prompts, in addition to basic information (name, email, and institution):

- In one paragraph, please describe what your research is about

- In one paragraph, please describe your plan for using CrowdTangle data to support your research

I and others who have applied have heard a response from CrowdTangle promptly that my application was approved. Graduate students are eligible to apply for CrowdTangle.

\section{Creating and Uploading a List of Pages to Crowd Tangle}

The next step assumes that your application has been approved. There are many ways to use CrowdTangle $^{3}$; here, we focus on using a list of pages to access historical data. We use a list of pages because doing so allows one to identify multiple pages - even hundreds or thousands of pages - and to download posts from those pages in a single step.

How do you identify the pages to add to a list? In past research, I web scraped the pages — accessed the pages using code — of every United States' school district's homepage, identifying links to a Facebook page. Pages can also be identified manually, from collections of pages identified by others and through searching with keywords through CrowdTangle itself.

For this tutorial, we have created a list of the 100 largest United States school districts' (based on the number of students enrolled in the district) Facebook pages. Notably, we were able

${ }^{2}$ The application is at this URL: https://help.crowdtangle.com/en/articles/4302208-crowdtanglefor-academics-and-researchers

${ }^{3}$ Resources for getting started with CrowdTangle

https://www.crowdtangle.com/resources/best practices 
to identify pages for all the districts, pointing to how widespread the use of Facebook by educational institutions such as schools or districts might be.

First, log in to CrowdTangle 4 . after logging into CrowdTangle, from the CrowdTangle homepage, click "+ Create List." Then click "For Pages." At the top of the page that opens, you will see a blank field for the list's name. Name this something short and easy to type; I used 100largest-school-districts for the name. After naming the list, you should see a mostly blank page. Now it is time to upload the list of pages. I used a Google Doc to create the list of pages ${ }^{5}$. Note that there must be two columns, and they must be named 1) Page or Account URL and 2) List. There can also be other columns with other information, but these two must be present. In the Page or Account URL are the URLs to the Facebook pages for the districts; this is - obviouslydifferent for each district. In the List column is the same thing for every district: it is the name of the list, 100-largest-school-districts; I copied and pasted this to be the same for every district.

Here are what the first ten districts look like in this spreadsheet; there are 100 districts in total in the spreadsheet (Figure 1).

\section{Figure 1.}

The first two columns and ten rows of the spreadsheet I created for the Facebook pages for the 100 largest school districts.

\begin{tabular}{l|l}
\hline Page or Account URL & List \\
\hline https://www.facebook.com/NYCschools & 100 -largest-school-districts \\
\hline https:/www.facebook.com/losangelesschools & 100 -largest-school-districts \\
\hline https:/www.facebook.com/chicagopublicschools & 100 -largest-school-districts \\
\hline https:/www.facebook.com/miamischools & 100 -largest-school-districts \\
\hline https:/www.facebook.com/clarkcountyschooldistrict & 100 -largest-school-districts \\
\hline https:/www.facebook.com/browardschools & 100 -largest-school-districts \\
\hline https:/www.facebook.com/hillsboroughsch & 100 -largest-school-districts \\
\hline https:/www.facebook.com/houstonisd & 100 -largest-school-districts \\
\hline https:/www.facebook.com/orangecountypublicschools & 100 -largest-school-districts \\
\hline https:/www.facebook.com/pbcsd & 100 -largest-school-districts \\
\hline
\end{tabular}

Next, in the top-right corner of the CrowdTangle homepage, click the gear symbol, then click "Batch Upload". This is where you will upload the spreadsheet with the list we have just created.

To do so, first, download the Google Sheet as a CSV - a comma-separated value file that CrowdTangle requires. Then, from the Batch Upload page, browse your computer to select this file, and click "Upload Now." You should see the message "Validating the CSV" and then a progress bar indicating that the file is being processed. After a few moments, you should see a message that the upload was complete. Navigate now to the list you just created; in this case, it is entitled 100-largest-school-districts. You should see the most recent posts by the pages you just uploaded, as below in Figure 2.

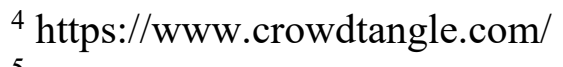

5

https://docs.google.com/spreadsheets/d/1Yu2O_geRKo9mRvrESplkiszUGSDbqVL8oLX_HDzo gmM/edit\#gid=0
} 
Figure 2.

The homepage for the list once the batch upload has been completed with one recent post displayed

100-largest-school-districts

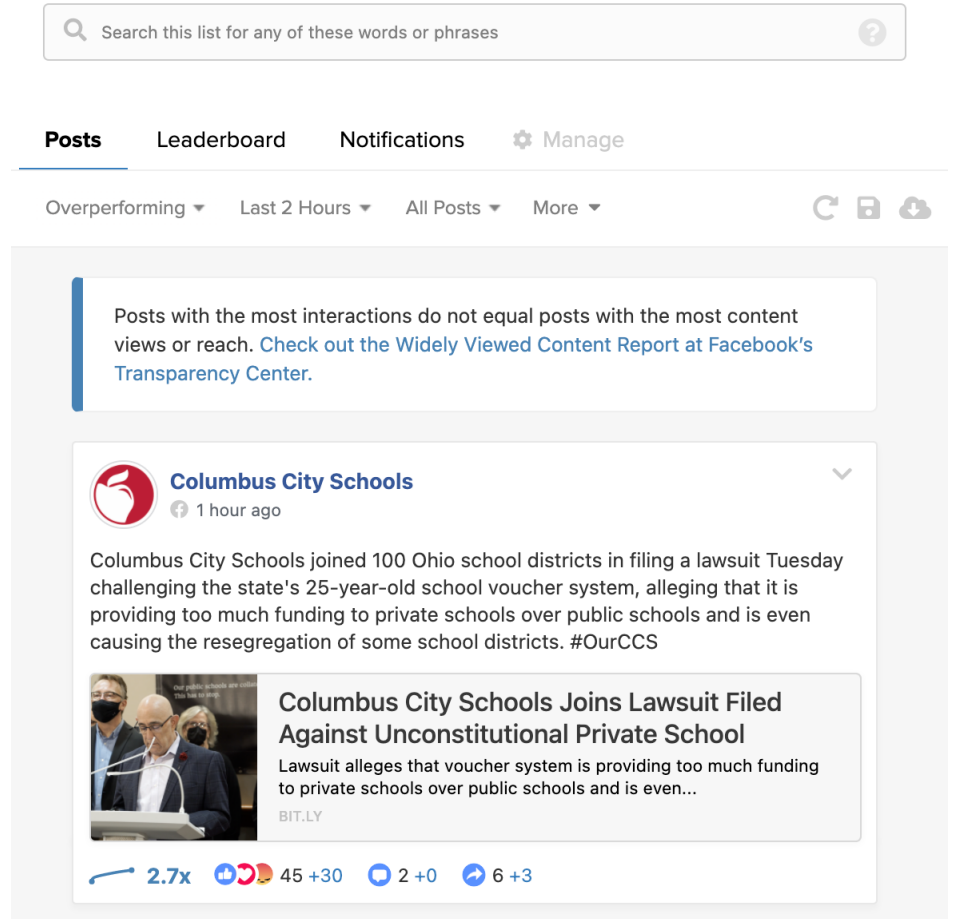

You may also notice the number of pages in your list. In this case, it is less than 100: it is 95. This is because five of the 100 pages we identified were ineligible to be accessed through CrowdTangle. Reviewing the email that CrowdTangle automatically sends following a batch upload, we can see that those that were not uploaded were not because "Producer doesn't meet the eligible criteria or doesn't exist." This likely means that those pages were private and therefore are not accessible via CrowdTangle.

At this point, we could carry out analyses within CrowdTangle. For instance, navigating to the "Leaderboard" for the 100-largest-school-districts list shows us some interesting statistics for the 95 pages. However, we can do much more with the data if we download it and then use other tools to analyze the data in the ways we see fit. I will describe how to do this in the next step.

\section{Downloading Historical Data Using CrowdTangle}

Having uploaded a list, we can now download historical data for any period for which there was activity by one or more pages. To do so, we can navigate again to the gear symbol in the top-right of the page, then to "Historical Data."

To download all of the information on the districts' posts during 2021, we select that we want to choose the 100-largest-school-districts as the list for the scope of the search; to not make any changes to filter what is returned in the section on the types of posts; and to specify January 1, 2021-December 31, 2021 as our date range. See Figure 3 for the parameters used for the query. 
Running head: FACEBOOK AS DATA

Figure 3.

Settings to download all the posts for 2021 for the pages on the 100-largest-school-districts list

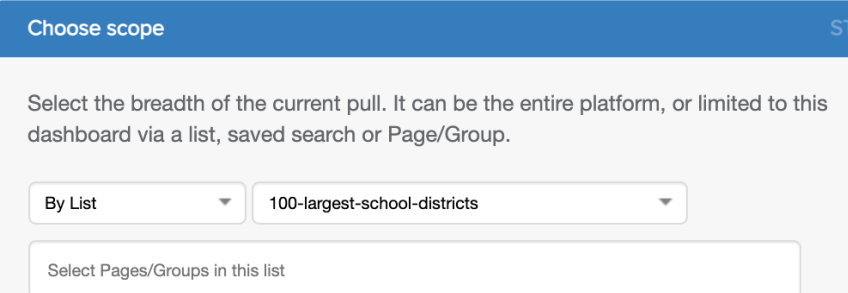

\section{Filter the types of posts}

Optionally, select a specific post type, add a search term, select order, and/or limit the number of posts returned.

Searc

Post Type

Order By

Limit Results

Any words or phrases (e.g. Trump, Chance the Rapper)

Branded Content Only

Marked As Breaking Only

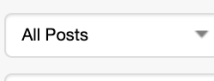

Most Recent

No limit

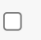

$\square$

\section{Pick your dates}

Choose from pre-selected timeframes or add your own custom date range.

Custom Date Range $\quad$

$01 / 01 / 2021$ to $12 / 31 / 2021$

We then click "Fetch History" at the bottom. CrowdTangle will send an email when the posts are ready to download - typically within a few minutes. Within your email from CrowdTangle, click the link to download a CSV file containing all the posts from the districts for the 2021 year. We can then replicate this step for the 2019 and 2020 years, changing the dates; accordingly, we must do this step as there are more than 100,000 posts per year- the limit for a single historical data request. Next, we will explore how to analyze this data to answer our two questions.

\section{Analyzing Data Using $R$}

We have now downloaded the CSV file. We can upload this to Google Sheets or open it with Microsoft Excel to inspect the data or-if we prefer-to analyze the data using these or other tools. In this primer, we will use R, a statistical software and programming language well suited for analyzing digital (and other large or even messy) sources of data. An introduction to $\mathrm{R}$ is beyond the scope of this primer. Still, interested readers may find chapters $5^{6}$ and $6^{7}$ of Estellado et al.'s (2020) Data Science in Education Using $R$ useful for getting started.

\footnotetext{
${ }^{6} \mathrm{https}: / /$ datascienceineducation.com/c05.html

${ }^{7} \mathrm{https} / / /$ datascienceineducation.com/c06.html
} 
When did districts first post about the COVID-19 pandemic? To answer this question, I:

1. Load required packages (add-ons to $\mathrm{R}$ )

2. Read (open) the data in $\mathrm{R}$ and join the three separate data sets (prepare it for analysis by processing the date variables

3. Use regular expressions to identify mentions of "covid" (with any capitalization)

4. Create a visualization of the mentions of COVID-19 by month

The code to replicate each of these steps is included at

https://osf.io/jhnrb/?view only=43f2da07eee14feb90fcb1755bbdbcfe. The result of these steps is presented in Figure 4. From this figure, we can see that the first mentions of COVID-19 wereas could be predicted - in March 2020. While COVID-19 was mentioned in the months since, it was not mentioned as frequently as during that month, though there were peaks - particularly around August 2021, when school was returning and there were ample concerns about variants of the virus. Extensions of this initial analysis could examine the content, tone, or type of post and how these varied from before to during the earlier and later stages of the pandemic.

\section{Figure 4}

Mentions of COVID-19 in districts' posts on Facebook from 2019-2022

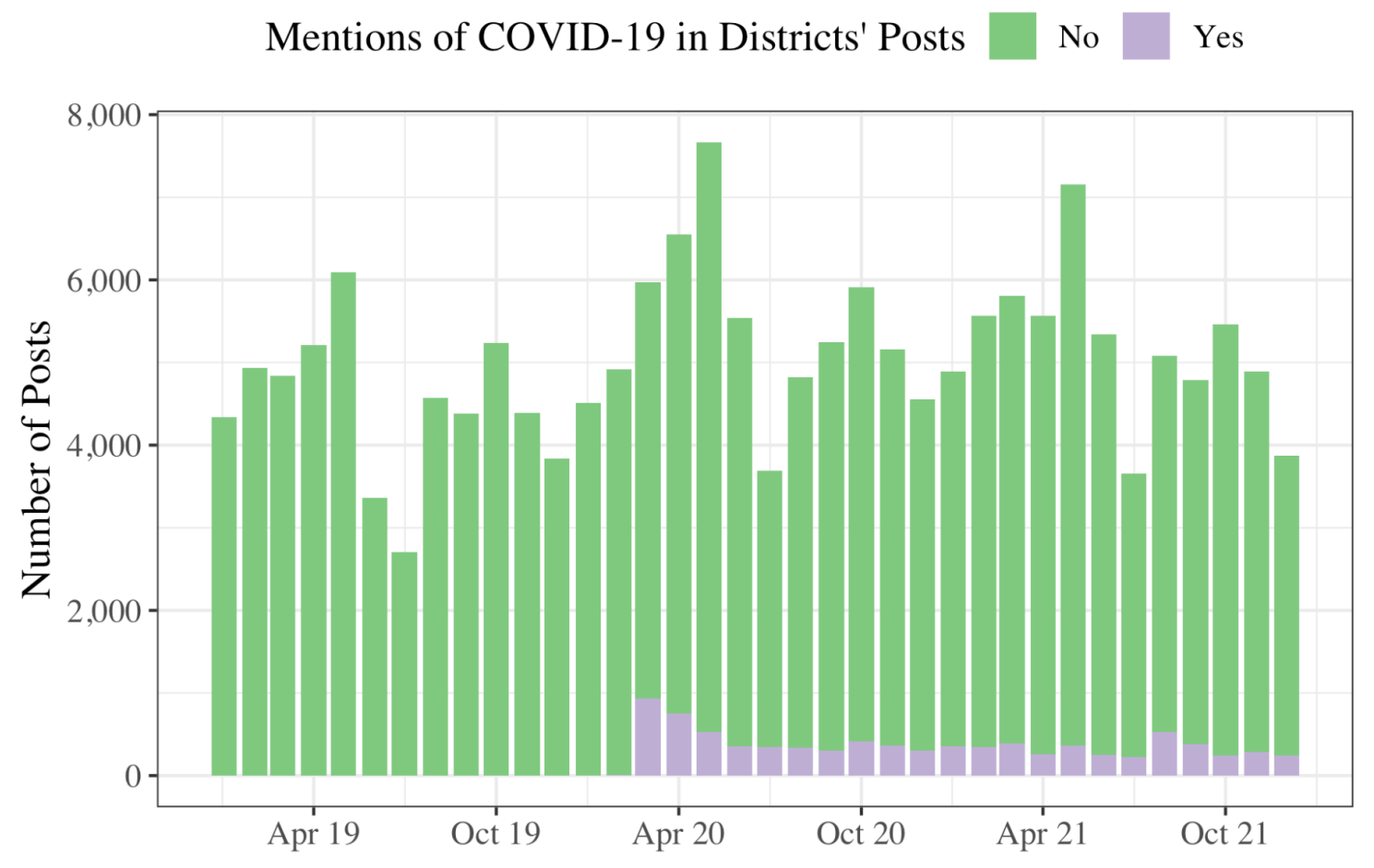

What relates to people interacting with districts' posts? To answer this question, we may skip the first two steps as those were already carried out for the above analysis; if the above 
analysis is not carried out, then steps 1 and 2s must be carried out first. Having loaded packages and the data, I:

5. Create summary statistics for a) total interactions (i.e., comments, likes, shares, "angry," etc.) and b) mean interactions per post, c) the year the page and determined, d) the number of followers of the district's page, and the size of the district (ranked within the top 100 largest districts based on the number of students enrolled).

6. Join these variables together into a single time

I present the results of this analysis for the ten largest districts below.

\section{Table 1}

Characteristics of the Facebook pages for the ten districts with the most total interactions

\begin{tabular}{|c|c|c|c|c|c|}
\hline District & $\begin{array}{c}\text { Total } \\
\text { Interacti } \\
\text { ons (3 } \\
\text { Years) }\end{array}$ & $\begin{array}{c}\text { Mean } \\
\text { Interacti } \\
\text { ons Per } \\
\text { Post }\end{array}$ & Page Created & $\begin{array}{c}\text { Follower } \\
\text { s }\end{array}$ & $\begin{array}{c}\text { Size of } \\
\text { District } \\
\text { (Rank) }\end{array}$ \\
\hline Pasco County Schools (FL) & 833,676 & 660.59 & 2012 & 59,803 & 48th \\
\hline $\begin{array}{l}\text { Mobile County Public } \\
\text { Schools (AL) }\end{array}$ & 746,151 & 319.96 & 2009 & 56,477 & 78th \\
\hline $\begin{array}{l}\text { North East Independent } \\
\text { School District (TX) }\end{array}$ & 720,586 & 161.85 & 2014 & 38,368 & $62 \mathrm{nd}$ \\
\hline Dallas ISD (TX) & 719,973 & 149.18 & 2010 & 84,468 & 16 th \\
\hline $\begin{array}{c}\text { Douglas County School } \\
\text { District }(\mathrm{CO})\end{array}$ & 631,237 & 226.08 & 2010 & 30,214 & 57 th \\
\hline Northside ISD (TX) & 610,842 & 279.94 & 2009 & 57,491 & $61 \mathrm{st}$ \\
\hline $\begin{array}{l}\text { Hillsborough County Public } \\
\text { Schools (FL) }\end{array}$ & 602,330 & 363.94 & 2012 & 68,076 & 7 th \\
\hline Fort Worth ISD (TX) & 507,828 & 94.95 & 2009 & 48,276 & 38th \\
\hline $\begin{array}{l}\text { Polk County Public Schools } \\
\text { (FL) }\end{array}$ & 503,935 & 213.35 & 2010 & 50,510 & 27 th \\
\hline $\begin{array}{l}\text { Anne Arundel County Public } \\
\text { Schools (MD) }\end{array}$ & 472,360 & $\begin{array}{l}148.9624 \\
72\end{array}$ & 2012 & 66,743 & 39th \\
\hline
\end{tabular}

Like for above, the code to replicate each of these steps is included in supplementary material A. These results show, first, that some districts receive many likes - posts by Pasco County Schools alone, for instance, have received nearly one million likes over the past three years; on average, their posts receive around 660 interactions. Notably, that district is not among the very largest in the nation - its size is 48th of the 100 largest districts. Moreover, apart from 
Hillsborough County Public Schools (also in Florida), most of the districts were not among the very largest, suggesting that interactions with districts' pages on Facebook are a function of factors other than the mere number of students (and students' parents) in the district. This suggests that future research could explore what factors - the type or content of the post, for instance, demographic information or how Facebook fits within the district's communication plan-help to explain these differences in interactions.

\section{Discussion}

At the outset, I motivated this paper based on the value of digital trace data for studying educational technology tools broadly, and social media platforms, specifically. I argued that there are opportunities to explore topics and events and people and organizations using digital data that can be accessed through Facebook. Using the posts of the 100 largest school districts to illustrate how Facebook data can be accessed and analyzed through CrowdTangle and R, respectively, this study illustrated one approach to the use of such data.

The pages I accessed should not necessarily be taken as a recommendation by others to study the posts of school districts. Research on Twitter has evolved in manifold ways, addressing a wide variety of topics that were probably difficult if not impossible to have planned to have addressed. For instance, how could researchers have planned to study a network that emerged in the days following a national tragedy (e.g., Greenhalgh \& Koehler, 2017)? Thus, in this section, I describe two general future directions for research on Facebook, adding as a caveat that these are merely suggestions based on some experience made with the understanding that there are many worthwhile ideas for studies that involve education-related Facebook data that are difficult to anticipate.

\section{Educational Technology Research With Facebook}

First, researchers may consider scale. Specifically, researchers may consider creating lists of pages other than the largest school districts in the United States-especially at a large scale, as the wide use of Facebook can enable large-scale analyses. This data source and others that require large-scale data collection and analysis may be fruitful to carry out using the CrowdTangle platform (and Facebook data). There are many education-related pages (and groups, which - if they are open - may also be accessed with CrowdTangle) beyond those of schools and districts that others may know about and use. Scale is especially relevant for considering what kinds of research should be carried out. Topics and events and people and organizations, particularly, may be studied in a particular way that takes advantage of the scale of social media data. There are examples of research on other platforms that do this (e.g., Carpenter et al., 2020; Staudt Willet, 2019; Staudt Willet \& Carpenter, 2020; Veletsianos \& Kimmons, 2016). At the same time, there is research on the analysis of more select sets of Facebook data. Research that takes advantage of the scale of social media might consider topics or events that span across multiple Facebook groups, different from past research that examined one (Lunden et al., 2020) or a few (Liljekvist et al., 2020; van Bommel et al., 2020) groups; otherwise, strictly manual, qualitative approaches are likely appropriate - not meriting the need to access the much more extensive data available through CrowdTangle.

Furthermore, research might consider examining people or organizations related to teaching, learning, and educational systems on Facebook. Past research on K-12 educational institutions has yielded several insights into the technologies schools and districts communicate about (Kimmons et al., 2021, 2022), for instance. Future research might investigate interactions with different posts from these and other educational institutions, such as early childhood institutions, post-secondary institutions, and the myriad organizations that support or advocate 


\section{Running head: FACEBOOK AS DATA}

for educators. Many such pages exist, and none I am aware of have been studied using the kinds of big data-specific approaches detailed here or using any other approaches. Furthermore, there are opportunities to study the content of such posts using linguistic techniques such as those used by Barnes (2021) — and used by Supovitz and Reinkordt (2017) in other (Common Core State Standards) contexts. Lastly, research on people (rather than organizations) is likely more challenging to carry out at scale, as the individuals of most individual people - including educators and educational leaders - are likely not public. Thus, Facebook data is fundamentally different from data from other platforms, especially Twitter, in that only public Facebook pages and groups are accessible to researchers.

Second, researchers should consider time. Historical access to data means that it is possible to "time travel" and to access data long after it is possible to-for instance — ask teachers what they thought of a specific topic (like a technology) or an event (like a change in policy). My colleagues and I carried out a very brief study to understand what schools and districts posted during the early stages of the COVID-19 pandemic (Rosenberg \& Nguyen, 2020). Like for scale, the ability to time travel may be a fruitful avenue for educational technology researchers to consider. What were districts posting in - for instance - a decade ago, in 2011, and what can such posts and where they take place tell us about teaching, learning, and educational systems? Again, this recommendation is not limited to schools' and districts' posts, but to think broadly about what may have been posted on a public page or group on Facebook and whether and how such posts could be useful from the vantage of educational technology research. For this and the above recommendation, what researchers know best-topics or events that may have taken place through or with specific people and organizations - may be good starting points for research.

\section{Limitations}

It would be a mistake not to mention some of the limitations of digital trace data in the context of Facebook. As Stier et al. (2020) note, using self-report and digital trace data are not in conflict: They lend complementary insights that can address the weaknesses of the other (Stier et al., 2020), and they are often used by the same researchers - even in the same studies (Al Baghal et al., 2020; Vraga \& Tully, 2020). But, while digital and self-report methods are not in opposition, the insights offered by studies using self-report and digital trace data are both complementary and different (Stier et al., 2020). Such insights can be garnered whether posts were one day or one year ago, allowing researchers to understand what was being posted long after the collection of self-report data is possible. Moreover, while self-report data are likely a better data source for understanding people's preferences, beliefs, and reflective thought, what people post can also reveal what they pay attention to, say, and amplify. This paper in no way is meant to imply that asking people (including educators) about how they use Facebook through self-report surveys is not likely to be a valuable activity: as a reviewsof past research on Facebook (e.g., Liljekvist et al., 2021) has illustrated, these studies are valuable. Moreover, selfreport data can complement the data accessed through Facebook. For instance, a longitudinal study of the posts by the page for an educational technology company from the first to the most recent post could be complemented by interviews with or surveys of the creators or users of that technology; both can illuminate different aspects of the role of the technology in education.

\section{Conclusion}

While this study provided the analyses of two research questions (using the posts on Facebook of the largest school districts as the source of data), I urge educational technology researchers to think broadly about the data sources, research questions, and analyses to be carried 
Running head: FACEBOOK AS DATA

out. A reflection on how other fields, like public health (e.g., Ayers et al., 2021) and information science and communication (Théro \& Vincent, 2022) are using data from the most widely-used social media platform suggests that there are many such opportunities for researchers in the educational technology field and in the adjacent fields of educational data mining and learning analytics. Just as creative research has invited new possibilities for teaching and learning using social media and other digital spaces, research that uses Facebook as a source of data can, too, while also highlighting drawbacks or negative aspects of this platform at the same time. 


\section{References}

Al Baghal, T., Sloan, L., Jessop, C., Williams, M. L., \& Burnap, P. (2020). Linking Twitter and survey data: The impact of survey mode and demographics on consent rates across three UK studies. Social Science Computer Review, 38(5), 517-532.

Ayers, J. W., Chu, B., Zhu, Z., Leas, E. C., Smith, D. M., Dredze, M., \& Broniatowski, D. A. (2021). Spread of misinformation about face masks and COVID-19 by automated software on Facebook. JAMA internal Medicine, 181(9), 1251-1253.

Barrot, J. S. (2021). Scientific mapping of social media in education: A Decade of Exponential Growth. Journal of Educational Computing Research, 59(4), 645-668. https://doi.org/10.1177/0735633120972010

Barnes, N. (2021). The social life of literacy education: How the 2018 \#phonicsdebate is reshaping the field. The Australian Educational Researcher. https://doi.org/10.1007/s13384-021-00451-x

Bodily, R., Leary, H., \& West, R. E. (2019). Research trends in instructional design and technology journals. British Journal of Educational Technology, 50(1), 64-79.

Bowman, N. D., \& Akcaoglu, M. (2014). "I see smart people!": Using Facebook to supplement cognitive and affective learning in the university mass lecture. The Internet and Higher Education, 23, 1-8.

Burchfield, M., Rosenberg, J., Borchers, C., Thomas, T., Gibbons, B., \& Fischer, C. (2021). In I.-H. Hsiao, S. Sahebi, F. Bouchet, \& J.-J. Vie (Eds), Proceedings of the 14th International Conference on Educational Data Mining (pp. 744-749). Are violations of student privacy "quick and easy"? Investigating the privacy of students' images and names in the context of K-12 educational institution's posts on Facebook. Proceedings of the 14th International Conference on Educational Data Mining (EDM).

Carpenter, J., Tani, T., Morrison, S., \& Keane, J. (2020). Exploring the landscape of educator professional activity on Twitter: An analysis of 16 education-related Twitter hashtags. Professional Development in Education, 1-22.

CrowdTangle Team (2021). CrowdTangle. Facebook, Menlo Park, California, United States. https://crowdtangle.com

Fischer, C., Pardos, Z. A., Baker, R. S., Williams, J. J., Smyth, P., Yu, R., ... \& Warschauer, M. (2020). Mining big data in education: Affordances and challenges. Review of Research in Education, 44(1), 130-160.

Gleason, B. (2016). New literacies practices of teenage Twitter users. Learning, Media and Technology, 41(1), 31-54.

Gobert, J. D., Sao Pedro, M., Raziuddin, J., \& Baker, R. S. (2013). From log files to assessment metrics: Measuring students' science inquiry skills using educational data mining. Journal of the Learning Sciences, 22(4), 521-563.

Gramlich, J. (2021). 10 facts about Americans and Facebook. https://www.pewresearch.org/facttank/2021/06/01/facts-about-americans-and-facebook/

Greenhalgh, S. P., \& Koehler, M. J. (2017). 28 Days Later: Twitter Hashtags as" Just in Time" Teacher Professional Development. TechTrends, 61(3), 273.

Greenhalgh, S. P., Koehler, M. J., Rosenberg, J. M., \& Willet, K. B. S. (2020). Considerations for using social media data in learning design and technology research. In Research methods in learning design and technology (pp. 64-77). Routledge.

Greenhow, C., \& Gleason, B. (2014). Social scholarship: Reconsidering scholarly practices in the age of social media. British Journal of Educational Technology, 45(3), 392-402. 
Greenhow, C., Galvin, S. M., Brandon, D. L., \& Askari, E. (2020). A Decade of research on K12 teaching and teacher learning with social media: Insights on the state of the field. Teachers College Record, 122(6), 1-72. https://doi.org/10.1177/016146812012200602

Hakimi, L., Eynon, R., \& Murphy, V. A. (2021). The ethics of using digital trace data in education: A thematic review of the research landscape. Review of Educational Research, https://journals.sagepub.com/doi/full/10.3102/00346543211020116

Hawksey, M. (2014). Need a better Twitter Archiving Google Sheet? TAGS v6.0 is here! [Blog post]. Retrieved from https://mashe.hawksey.info/2014/10/need-a-better-twitterarchiving-googlesheet-tags-v6-0-is-here

Heffernan, N. T., \& Heffernan, C. L. (2014). The ASSISTments ecosystem: Building a platform that brings scientists and teachers together for minimally invasive research on human learning and teaching. International Journal of Artificial Intelligence in Education, 24(4), 470-497.

Kennewell, S. (2001) Using affordances and constraints to evaluate the use of information and communications technology in teaching and learning, Journal of Information Techology for Teacher Education, 10, 1-2, 101-116.

Kimmons, R., \& Veletsianos, G. (2018). Public internet data mining methods in instructional design, educational technology, and online learning research. TechTrends, 62(5), 492500 .

Kimmons, R., Rosenberg, J., \& Allman, B. (2021). Trends in educational technology: What Facebook, Twitter, and Scopus can tell us about current research and practice. TechTrends, 1-12.

Kimmons, R., Rosenberg, J., \& Allman, B. (2021). Trends and Topics in Educational Technology, 2022 Edition. TechTrends. https://ink.springer.com/article/10.1007/s11528022-00713-0

Krutka, D. G., Heath, M. K., \& Willet, K. B. S. (2019). Foregrounding technoethics: Toward critical perspectives in technology and teacher education. Journal of Technology and Teacher Education, 27(4), 555-574.

Liljekvist, Y. E., Randahl, A. C., van Bommel, J., \& Olin-Scheller, C. (2021). Facebook for professional development: Pedagogical content knowledge in the centre of teachers' online communities. Scandinavian Journal of Educational Research, 65(5), 723-735.

Lundin, M., Lantz-Andersson, A., \& Hillman, T. (2020). Teachers' Identity Work in a Professional Facebook Group. Journal of Information Technology Research, 19, 205222. https://doi.org/10.28945/4540

Niu, L. (2019). Using Facebook for academic purposes: Current literature and directions for future research. Journal of Educational Computing Research, 56(8), 1384-1406.

R Core Team (2021). R: A language and environment for statistical computing. R Foundation for Statistical Computing. https://www.R-project.org/.

Romero-Hall, E., Kimmons, R., \& Veletsianos, G. (2018). Social media use by instructional design departments. Australasian Journal of Educational Technology, 34(5).

Rosenberg, J. M., Reid, J. W., Dyer, E. B., Koehler, M. J., Fischer, C., \& McKenna, T. J. (2020). Idle chatter or compelling conversation? The potential of the social media-based \#NGSSchat network for supporting science education reform efforts. Journal of Research in Science Teaching, 57(9), 1322-1355. 
Rosenberg, J. M., \& Nguyen, H. (2021). How K-12 school districts communicated during the COVID-19 pandemic: A study using Facebook data. In Proceedings of the 11th International Conference on Learning Analytics \& Knowledge (pp. 1-4).

Siemens, G., \& Baker, R. S. D. (2012, April). Learning analytics and educational data mining: towards communication and collaboration. In Proceedings of the 2nd international conference on learning analytics and knowledge (pp. 252-254).

Staudt Willet, K. B., \& Carpenter, J. P. (2020). Teachers on Reddit? Exploring contributions and interactions in four teaching-related subreddits. Journal of Research on Technology in Education, 52(2), 216-233.

Staudt Willet, K. B. (2019). Revisiting how and why educators use Twitter: Tweet types and purposes in\# Edchat. Journal of Research on Technology in Education, 51(3), 273-289.

Stewart, E. (2019). Facebook is sharing data to figure out how it messes with democracy. https://www.vox.com/recode/2019/5/1/18525316/facebook-research-data-democracycambridge-analytica

Stier, S., Breuer, J., Siegers, P., \& Thorson, K. (2020). Integrating survey data and digital trace data: Key issues in developing an emerging field. Social Science Computer Review, 38(5), 503-516.

Supovitz, J., \& Reinkordt, E. (2017). Keep your eye on the metaphor: The framing of the Common Core on Twitter. Education Policy Analysis Archives, 25(30), 1-29.

Théro, H., \& Vincent, E. M. (2022). Investigating Facebook's interventions against accounts that repeatedly share misinformation. Information Processing \& Management, 59(2), 102804.

Trust, T., Krutka, D. G., \& Carpenter, J. P. (2016). "Together we are better": Professional learning networks for teachers. Computers \& Education, 102, 15-34.

Tufekci, Z. (2014). Big questions for social media big data: Representativeness, validity and other methodological pitfalls. In the Eighth International AAAI Conference on Weblogs and Social Media.

van Bommel, J., Randahl, A. C., Liljekvist, Y., \& Ruthven, K. (2020). Tracing teachers' transformation of knowledge in social media. Teaching and Teacher Education, 87, 102958.

Veletsianos, G., \& Kimmons, R. (2016). Scholars in an increasingly open and digital world: How do education professors and students use Twitter? The Internet and Higher Education, $30,1-10$.

Vraga, E. K., \& Tully, M. (2020). Who is exposed to news? It depends on how you measure: Examining self-reported versus behavioral news exposure measures. Social Science Computer Review, 38(5), 550-566.

Watson, S., \& Barnes, N. (2021). Online educational populism and New Right 2.0 in Australia and England. Globalisation, Societies and Education, 1-13. https://doi.org/10.1080/14767724.2021.1882292

Watters, A. (2014). The monsters of education technology. CreateSpace.

Wise, A. F. (2019). Learning analytics: Using data-informed decision-making to improve teaching and learning. In Contemporary technologies in education (pp. 119-143). Palgrave Macmillan, Cham. 